\title{
Investigating origin of the inadequate medium range predictability of the lower tropospheric ultra-long waves in tropics
}

\author{
S DE \\ Indian Institute of Tropical Meteorology, Dr. Homi Bhabha Road, Pashan, Pune 411 008, Maharastra, India. \\ e-mail: sde@tropmet.res.in
}

The predictability of planetary or ultra-long scale waves is limited by the large growth of errors in these scales in almost all the medium range forecast models. Understanding the cause for the enormous build up of error is, therefore, a necessary task for improving the prediction of planetary waves. A diagnostic analysis of the systematic error energetics has been performed in the Global Forecast System model to investigate the reasons for poor predictability of the lower tropospheric ultra-long waves (wavenumber bands 1-4) in tropics using the analysis-forecast system of horizontal wind field at $850 \mathrm{hPa}$ level during the boreal summer period. For this purpose, systematic error energy is computed in spatial as well as in wavenumber domain. Non-linear inter-scale transfer of error has been formulated and evaluated through energy exchanges among participating triads. The study reveals that the error is generated in the prognostic model initially with a small magnitude at the different locations around tropical convergence zone (TCZ) attributed to the inaccuracy in representing different physical processes like cumulus convection applied in the model. At subsequent evolution of forecasts, error increases and spreads along the TCZ due to its non-linear advection to the higher scales and eventually to the ultra-long scales attributed to the inherent dynamics of the model evaluated through the process of wave-wave exchange of error energy in terms of the triad interactions. The continuous generation and then, non-linear propagation of error up to the planetary scales in the course of prediction increase the uncertainty in ultra-long scales which actually inhibit to predict accurately the planetary scale waves in tropics during medium range forecasts. This work suggests caveats to the modeler's community in the predictability study of tropical ultra-long waves.

\section{Introduction}

One of the most well-known aspects in the behaviour of the ultra-long waves of wavenumbers $1-4$ is that its actual forecast deviates from the predictability theory expectations. In theory, the planetary waves are documented in the initial conditions and as the forecast error contaminates first the smallest scale and is transferred nonlinearly to the lower wavenumber it is expected that the planetary scale waves ought to display larger forecast skill compared to any shorter wavelength at any forecast period (predictability theory was elucidated in Lorenz's (1969) paper). But, in actual practice, there is a great difficulty observed in predicting the ultra-long waves in numerical weather prediction particularly in the first few days of the forecast period (Baumhefner et al 1978; Lambert and Merilees 1978).

The predictability of ultra-long waves has been a cause of major concern for the medium range weather prediction community for quite some time. The issue was addressed by Gall et al (1979) and Somerville (1980) for the mid-latitude region.

Keywords. Systematic error energetics; scale interactions; triads; wave-wave exchange of error energy. 
Gall et al (1979) examined the influence of the cyclone scale forcing on the mid-latitude ultra-long waves (zonal wavenumbers 1-4) with a numerical experiment carried out with simplified GCM. They concluded that the inter-scale interaction must be taken into account in the energy estimation of the ultra-long waves as the planetary scale error was evolved not only from the model deficiency but was partially due to the error in cyclone scale also. Somerville (1980) in his paper improved the understanding of the dynamics of the mid-latitude ultra-long waves (zonal wavenumbers 1-3) evaluating the effect of the tropical data on the predictability of the waves. The author admitted the complicacy in predicting the mid-latitude planetary waves due to many poorly understandable phenomena like the synoptic scale influence on the large scale through the process of scale interactions. He concluded that the evolution of the ultralong waves in the predictability theory aspects was of great theoretical as well as practical importance. Lucarini et al (2007) inter-compared the statistical properties of the model biases with a view to the initial quality check up of the climate model among 19 model members of Inter Governmental Panel on Climate Change (IPCC) with respect to the National Centre for Environmental Prediction (NCEP) and European Centre for Medium Range Weather Forecast (ECMWF) analysis utilizing Hayashi's (1979) spectra of mid-latitude $500 \mathrm{hPa}$ geopotential height. The inability of the dynamical models in predicting intra-seasonal oscillations (ISOs) (being in the range of ultra-long scale of wavenumbers 1-4) has been partly related to the models systematic bias in simulating the spacetime structure of the ISOs and partly due to upscale spreading of errors from small to large scales (Krishnamurti et al 1990). But the explicit mechanism of the non-linear upscale propagation of error has not been revealed in Krishnamurti et al's (1990) paper.

The difficulty in dynamical medium range forecast of tropical planetary scale waves has not so far been explicitly addressed. Lambert et al (1978) studied the 96-hour forecast error spectra in spherical harmonics and showed that the largest amplitude of the harmonics corresponding to the zonal wavenumbers 1 and 2 and their two dimensional part appeared over tropics. Kanamitsu (1985) has observed that the tropical large scale error is generally associated with wavenumber 1 . Deficiency in cumulus parameterization process may play a major role for the generation of the large scale tropical systematic error at lower tropospheric level (Heckley 1985; Kanamitsu 1985). Moreover, Majda and Biello (2004); Majda and Stechmann (2009) suggested that insufficient representation of planetary scale ISOs by the general circulation model is due to the inadequate treatment across multiple scale interactions of organized tropical convection. As the errors in the planetary wave structure are consistently growing (figure 1), from the findings of Majda and Biello (2004) and Majda and Stechmann (2009), the insufficient formulation of different physical processes such as the organized tropical convection would not simply answer the causes of poor predictability of the tropical ultra-long waves in weather prediction models unless the multiple scale interactions are adequately evaluated. Then, what theory could account for the mechanism by which scale interactions inhibit the predictability of planetary scale waves? The objective of the paper is to understand the role of scale interactions in error growth of the lower tropospheric planetary scale waves which limit the forecast skill of these waves in medium range tropical weather prediction model.

This objective is further necessitated by Straus and Shukla (2005). They commented that the growth rates of the tropical medium range forecast errors were invariant since the last decade. Only the improvement of the tropical forecast beyond 1-day lead was achieved by lowering the first day error. So, a general conceptual understanding of the error and the nature of the error growth in the tropics are very much relevant. Moreover, some of the major science focuses of THORPEX (The observing system research and predictability experiment) established in 2003 by the World Meteorological Organization as a 10 years international global atmospheric research and development program are model error, error dynamics and scale interactions. With this view, the following questions may be raised in the backdrop of error growth at the lower tropospheric planetary scale waves over the tropical region.

- How does the systematic error, generated with a small magnitude and confined into the small regions at the initial forecast period, converge ultimately with a large magnitude into the wide region of planetary scale tropical convergence zone (figure 1)?

- What is the nature of the propagation of the error variance across scales and of the scale interactions over the tropical region? Is there any inter-scale influence on the planetary scale error growth?

- What is the error dynamics of the ultra-long waves in tropics?

The quest for answers of the above questions through this paper may shed light on the growth of the uncertainty in the tropical planetary scale waves during the medium range forecasts. 


First question will be dealt through a careful depiction of the spatial and wavenumber distribution of the systematic error kinetic energy as the systematic error is generally associated with the planetary scale (Dalcher and Kalaney 1978; Kanamitsu 1985). It may be visualized by the spectral analysis that how the systematic error propagates across scales and in which direction (upscale or downscale) with the increase of the forecast time. To get insight into the possible answer of the second question mentioned above, author wishes to explore the scale interactions computing the wavewave exchanges of the error energy among different waves. This wave-wave exchange may be evaluated by resolving the dominant spectral mode into individual triad interactions associated with the different dynamical processes in the error growth rate equation following De and Chakraborty (2004). As far as the last question is concerned, the explicit investigation of the error characteristics in above may establish the science of error dynamics of the tropical planetary scale waves.

Triad interaction is essentially a unique mathematical tool to measure the wave-wave exchange applying the Fourier method. It was first formulated by Saltzman (1957) and later the physical explanation of this method was given by Murakami and Tomatsu (1964) and Tomatsu (1979). This mathematical instrument invokes the inter-scale information in the form of the nonlinear energy exchange into individual triad interactions that contribute to the total exchange associated with any particular scale whose triad interaction is to be computed. Thus the effect of one scale to other scale may also be known. The device was extensively used by Krishnamurti et al (2003); Krishnamurti and Chakraborty (2006) to delineate the nonlinear aspects of the intraseasonal oscillation. The formalism of the triad interaction entails the Fourier series of nonlinearities associated with the triple product term. Basic formulations of error kinetic energy, its growth rate and wave-wave exchanges through triple product nonlinearity are described in section 2. Model and data used for the diagnostic study is noted in section 3. Results are discussed in section 4 and conclusions are summarized in section 5 .

\section{Formulations related to the error kinetic energy}

\subsection{Systematic error energy and its growth rate equations}

The systematic error in the energy/variance form (hereafter referred to as the systematic error energy/variance), which is the function of forecast

time/lead $(t)$ only, may be obtained following Boer (1993) as:

$$
K^{s}(t)=\overline{\frac{1}{2} V_{e}^{s} \bullet V_{e}^{s}}
$$

where $V_{e}^{s} \equiv\left(u_{e}^{s}, v_{e}^{s}\right)$ is the part of the total error (difference between forecast and analyzed fields) obtained by ensemble averaging over many forecasts of the horizontal wind field at the same lead. $u_{e}^{s}$ and $v_{e}^{s}$ represent the same but for zonal and meridional wind component, respectively. The big dot shows the dot product and the overbar represents the spatial average. The systematic error energy growth rate equation in a spatially averaged form (Boer 1993; De and Chakraborty 2007) is expressed as:

$$
\begin{aligned}
\frac{\partial K^{s}(t)}{\partial t}= & -\nabla \bullet\left[\overline{\left(\frac{V_{e}^{s} \bullet V_{e}^{s}}{2}+V_{e}^{s} \bullet V_{e}^{r}\right) \cdot V_{f}}\right] \\
& +\left[\overline{\left.\frac{\left(u_{e}^{r} \cdot V_{o}+u_{e}^{r} \cdot V_{e}^{r}\right) \bullet \nabla u_{e}^{s}}{+\left(v_{e}^{r} \cdot V_{o}+v_{e}^{r} \cdot V_{e}^{r}\right) \bullet \nabla v_{e}^{s}}\right]}\right. \\
& -\left[\overline{\left(\frac{\left.u_{e}^{s} \cdot V_{e}^{s} \bullet \nabla u_{o}+v_{e}^{s} \cdot V_{e}^{s} \bullet \nabla v_{o}\right)}{+\left(u_{e}^{s} \cdot V_{e}^{r} \bullet \nabla u_{o}+v_{e}^{s} \cdot V_{e}^{r} \bullet \nabla v_{o}\right)}\right]}\right. \\
& +\overline{V_{e}^{s} \bullet R_{s}}
\end{aligned}
$$

where $V_{e}^{r} \equiv\left(u_{e}^{r}, v_{e}^{r}\right)$ is the random error that is another part of total error represented as the deviation from systematic error at each day for a fixed forecast lead. $u_{e}^{r}$ and $v_{e}^{r}$ are the random errors in zonal and meridional wind, respectively. The subscripts $o$ and $f$ represent the observed and forecast part of the wind field, respectively. The left hand side of the equation (2) is the growth rate term. The first term of the right hand side of equation (2) within the square bracket represents the nonlinear convergence or divergence of the flux of error energy. The second term evaluates the nonlinear conversion between systematic and random error. Similarly, the 3rd term estimates the nonlinear generation of systematic error and the fourth term is the residual term which represents source/sink of error due to all other processes (like baroclinicity, dissipation, computational error, etc.) except the error for the above inertial processes (Boer 1993). Boer used the growth rate equation to evaluate the extratropical errors. Hence the equation was geostrophic and the basic variable was $500 \mathrm{hPa}$ height fields. 
For suitable application of the same equation in tropics author has considered the total wind field in terms of the basic variable in equation (2) as the equation is no longer geostrophic.

Utilizing the Fourier transform on equation (1), the systematic error kinetic energy in wavenumber domain may be shown as:

$$
K_{k}^{s}(t)=\overline{\frac{1}{2}\left(u_{e}^{s} C_{k}^{2}+u_{e}^{s} S_{k}^{2}+v_{e}^{s} C_{k}^{2}+v_{e}^{s} S_{k}^{2}\right)}
$$

$C$ and $S$ in the right hand side terms of equation (3) represent the cosine and sine component, respectively and subscript $k$ stands for wavenumber.

\subsection{Computation of the wave-wave exchanges through triple product nonlinearities}

The three waves of different wavenumbers $k, m$ and $n$ in a triad $(k, m, n)$ interact if and only if they are inter-related with one of the following conditions $k=m+n, k=m-n$ and $k=n-m$ using the orthogonal property in trigonometry. These conditions are the so called trigonometric selection rule (Saltzman 1957). Here the wavenumber $k$ represents the wave whose triad interactions are to be computed while interacting with waves of wavenumbers $m$ and $n$. The different dynamical terms like flux, generation and conversion are shown as the sum of the product of three variables in equation (2). Now, applying the Fourier transform on each triple product term following Krishnamurti's papers $(2003,2006)$, the triad interactive form of flux, generation and conversion are constructed. As an example, the systematic error flux written as:

$$
F^{s}(t)=-\nabla \bullet\left[\overline{\left(\frac{V_{e}^{s} \bullet V_{e}^{s}}{2}+V_{e}^{s} \bullet V_{e}^{r}\right) \cdot V_{f}}\right]
$$

(taken from equation 2) is shown in terms of individual triad interaction in an Appendix. The spectrum of any dynamical term at wavenumber $(k)$ is evaluated adding all possible triad interactions of the wavenumber $k$ with other wavenumbers $m$ and $n$ computed from the triad interactive derivations of the term satisfying the selection rule. Thus, the whole spectra of different dynamical terms can be constructed.

\section{GFS model and data for the diagnostic study}

Diagnostic study has been performed on the analysis-forecast system of horizontal wind field of the Medium Range Weather Forecast (MRF) model of NCEP, now called the Global Forecast System (hereafter named as the GFS model).

The resolution of the GFS model is T126 having 28 unevenly spaced vertical levels. The model physics include long- and short-wave radiation with diurnal variation, cloud radiation interaction, planetary boundary layer processes, deep and shallow convection, large scale condensation, gravity wave drag, enhanced orography, simple hydrology and vertical and horizontal diffusion. Boundary layer effect are typically felt at the first five levels above the surface, i.e., at sigma $=0.995$, $0.981,0.960,0.920$ and 0.856 (Kanamitsu and Saha 1995).

The study has been performed with three different sets of data. First dataset contains daily analyzed and 1-7 day forecasts of horizontal wind field of $00 \mathrm{GMT}$ at $850 \mathrm{hPa}$ over $2.5^{\circ} \times 2.5^{\circ}$ grid size for summer (June-August) months having 100 days of data from 1 June to 8 September 2001. Similarly the second set of data comprises of 128 days daily data up to 8-day forecasts for the months of June, July, August and September having the same format with the first dataset but from 1 June to 6 October 2003. The specification of the third dataset is same as that of the 2nd set of data except for the year of 2004. All data have been downloaded from GFS site http://nomad5.ncep.noaa.gov/cgibin/ftp2u_mrf.sh. The data are chosen purely on random basis subject to the availability of continuous daily data in the GFS site. In the above dataset, month of September 2001 is not considered as couple of days data are not uploaded in the above site.

\section{Results and discussions}

The study has been discussed in two sections. In the first section the geographical distribution of the systematic error is described at $850 \mathrm{hPa}$ level with a view to investigate the possible initial source of forecast error in the lower tropospheric ultra-long waves over tropics. Other section deals with the spectral analysis of the error energy and its growth rate in order to find out the upscale propagation of the initial error up to the planetary scale and the large growth of the ultra-long scale error with the increase of the lead time. The role of multiple scale interactions in inter-scale error transfer will also be examined for the spectra of the dynamical terms (right hand side of the equation 2) in terms of the wave-wave exchange of error energy associated with dominant triad interactions. 


\subsection{Analysis in physical domain}

The systematic error energy is computed from the equation (1) at each grid point for 1-day to 7-day forecast period during 2001 and 1-8 day forecasts during 2003 and 2004. The error distribution is shown in figure 1 for $1,3,5$ and 7 -day lead during the northern hemispheric summer (June, July and August) 2001 and (June, July, August and September) 2003 and 2004 over the global tropics $\left(30^{\circ} \mathrm{S}-30^{\circ} \mathrm{N}\right)$. The error shown at each grid point is generated from the cumulative effect of the deficiencies in the different physical processes of the model. It is very difficult to evaluate the error attributed to any individual process. Only the significant cause is to be revealed for the generation of the systematic error at certain geographical locations. The figure shows that small amount of systematic error energy is appeared at some pockets around the region of TCZ at the initial forecast period up to 3 days for all the years. Gradually, the error is organized with increasing magnitude in the scale of TCZ that is the ultra-long scale over tropics. The region of maximum error is the tropical zone that is strongly associated with high cyclonic vorticity and convergence during the boreal summer (Sikka and Gadgil 1980). As the systematic error is defined as the error generated due to the deficiency of different physical processes described in the model (Boer 1993), the spatial distribution of the error shown in figure represents the area of strong convection where the cumulus parameterization may play a sensitive role in the presence of other model physics (Heckley 1985; Kanamitsu 1985). In view of the boundary layer process, friction is less active over ocean compared to that over land although the systematic error energy band is large over ocean. Moreover, model configuration shows that the boundary layer physics is critical upto the vertical level of sigma 0.856 . Therefore, boundary layer parameterization may not play a significant role in the generation of the systematic error at $850 \mathrm{hPa}$ GFS wind field over the region of study during the boreal summer period. Inadequate representation of various physical processes may initiate error in the model during the initial forecasts but what makes the successive build-up of error at the ultra-long scale (TCZ) with the increase of lead time shown in figure 1?

\subsection{Analysis in wavenumber domain}

The wavenumber spectra of the systematic error energy are computed from the equation (3) to investigate the growth of the planetary scale error. Figure 2 describes the wavenumber spectra of the systematic error in 2001, 2003 and 2004. The spectral distribution has shown an exponential curve
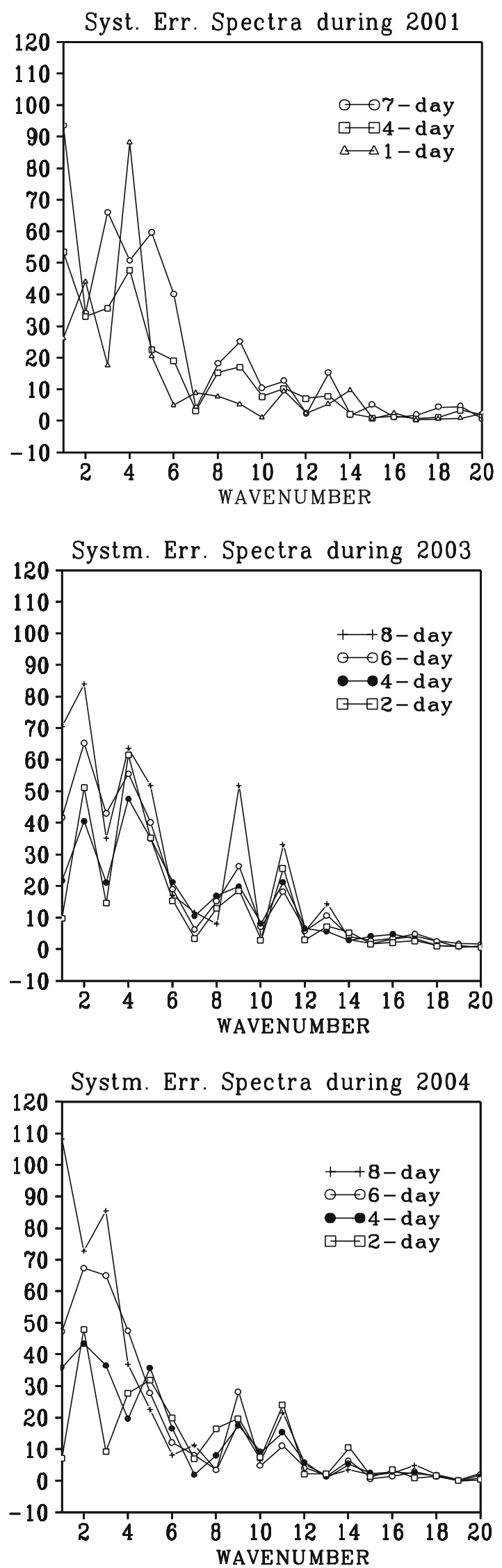

Figure 2. Spectral representation of the systematic error variance $\left(\times 10^{3}\right)$ of $850 \mathrm{hPa}$ GFS wind field for JJA, 2001 and JJAS of 2003 and 2004 in $\mathrm{m}^{2} / \mathrm{s}^{2}$ at different forecast period over $30^{\circ} \mathrm{S}-30^{\circ} \mathrm{N}$ tropical belt. 


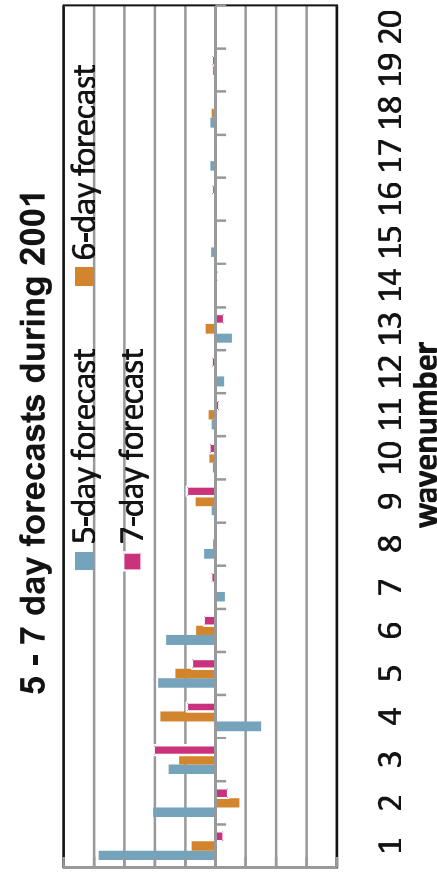

윢우윰유웅유윰우

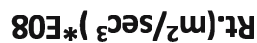

'Ч

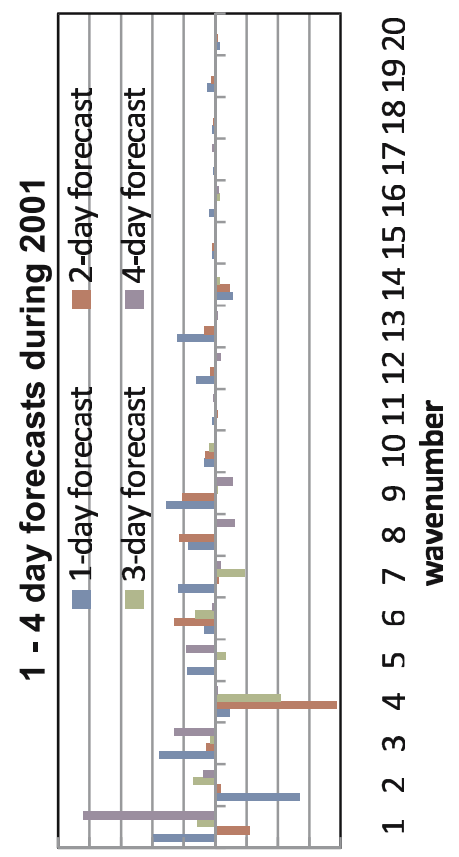

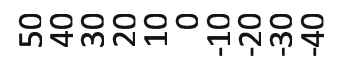

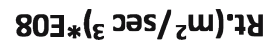

"Ч
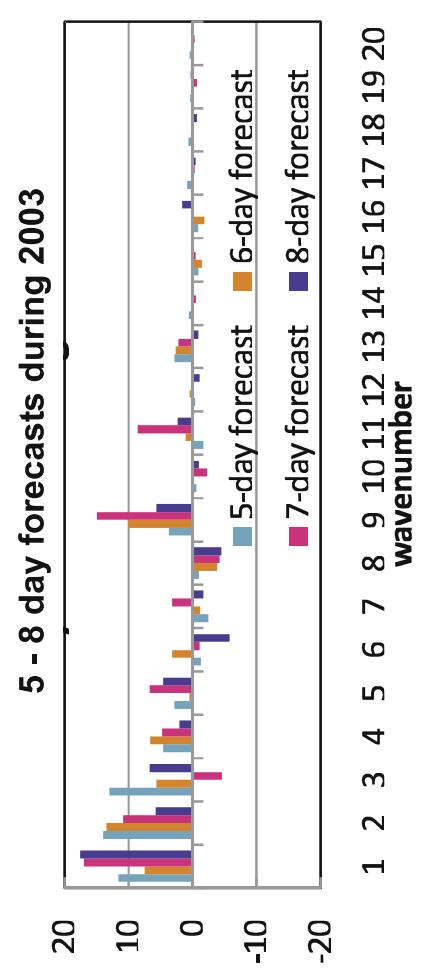

$803 *(\varepsilon \partial \partial \mathrm{s} / \mathrm{z} \mathrm{w}) \cdot 1 \mathrm{y}$

प

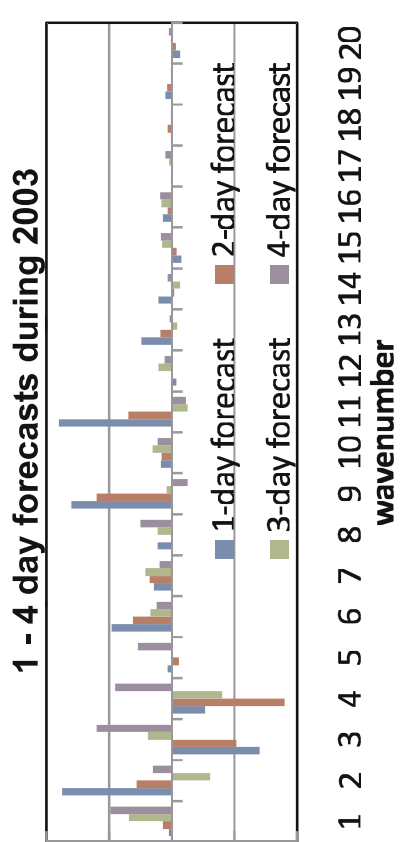

으 응 욱 $803 *(\varepsilon$ כəs $/ 2 \mathrm{~m}) \cdot 7 \mathrm{y}$

$479 \cdot 2 \cdot 13$

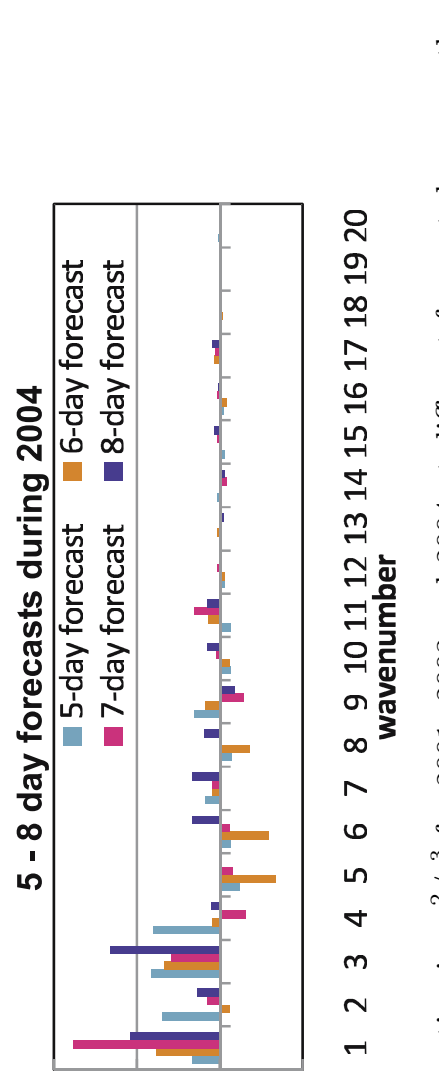

ㅇำ

$807 *\left(\varepsilon^{\partial a s} / \tau^{m}\right) \cdot 7 \mathrm{y}$

' ב

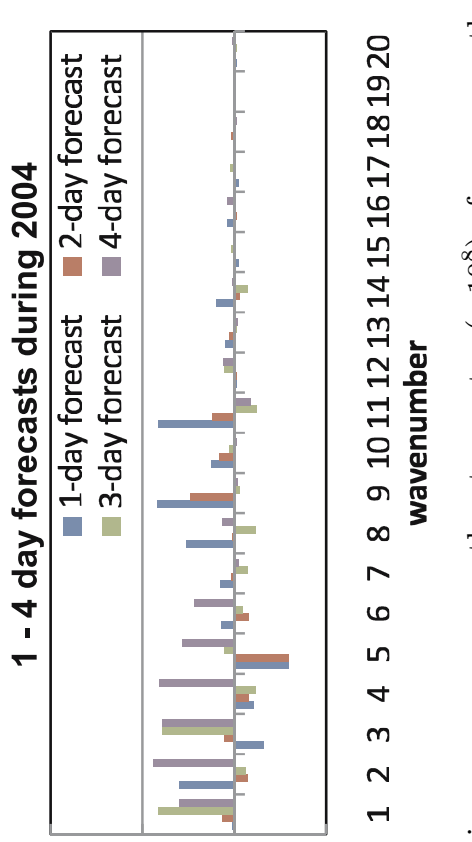

\& ㄱำ 위

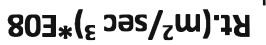

"प79. 
with the systematic development of error energy at the ultra-long scales of wavenumber range 1-4 with the increase of the forecast time. The error growth rate spectra are observed in figure 3 for all the years. The first panel depicts the nature of growth rate at the initial lead up to 4-day, whereas the second panel shows the same for the lead time up to 7 or 8 days. The figure reveals that the growth rate spectrum shows positive value at maximum wavenumbers up to 11 for the initial 4-day forecasts. As the forecast period increases the growth rates also increase to the large waves with its maximum value appeared at ultra-long scale waves. On the contrary, the growth rate decreases in the spectral region greater than wavenumber 5 and it shows small negative value at some wavenumbers during 2003 and 2004 in 5-8 day forecasts. This implies that systematic error grows almost equally up to wavenumber 11 at 1-4 day forecast period. Gradually, it grows more in the higher scales in comparison to the smaller scales. Moreover, there is an evidence of small dissipation of error at the shorter waves during 5-8 day leads. These phenomena support the fact that there may be spectral error propagation from small to the large scales. The insignificant growth rate of the waves greater than the wavenumber 11 is due to the small error energy capacity of these waves shown in figure 2 . So, it is inferred from the systematic error and its growth rate curves that the error may be transported upscale nonlinearly across the spectra with the increase of forecast lead.

The objectives of the spectral analysis of the inertial terms in the error growth rate equation are to show how the dynamical processes like flux, generation and conversion contribute to the growth of the planetary scale error; to choose the significant modes for triad interaction in the spectra of the dominant dynamical terms and to unravel the mechanism of non-linear upscale error transport. Following Boer (1984, 1993), it is required to examine the relative importance of each inertial term with respect to the residual in error energy growth rate budget (right hand side of equation 2) that will determine the significant dynamical processes for each year. For this purpose, the three geographical locations (India and its adjoining area, Equatorial West and East Pacific) along TCZ of the maximum systematic error obtained from figure 1 are exhibited by $10^{\circ} \times 10^{\circ}$ boxes and marked by 1,2 and 3 for every year shown in figure 4(a), 4(e) and 4(i). The dynamical and residual terms in all forecast days are represented as the line graphs averaged over each box shown in figure $4(\mathrm{~b}, \mathrm{c}, \mathrm{d}), 4(\mathrm{f}, \mathrm{g}, \mathrm{h})$ and $4(\mathrm{j}, \mathrm{k}, \mathrm{l})$ during 2001, 2003 and 2004, respectively. The absolute magnitude of each term in comparison to that of the residual at every lead measures the significance of that term with respect to residual in error growth rate budget. It has been revealed from figure that the error energy flux shows dominant with respect to residual for most of the forecast days in comparison to the conversion and generation terms in 2001 and 2004 analyzing figure $4(\mathrm{~b}, \mathrm{c}, \mathrm{d})$ and $4(\mathrm{j}, \mathrm{k}, \mathrm{l})$, respectively. Whereas nonlinear conversion (figure 4g) and flux (figure 4h) both are taken into account as they show large absolute magnitude compared to the residual in the year 2003. Hence the spectral analysis will be performed on nonlinear convergence and divergence of error energy flux for all the years and on nonlinear conversion between systematic and random error during the year of 2003 only. It may be noted that all the right hand side terms of equation (2) have shown the small magnitude at different leads in figures $4(\mathrm{f})$ and $4(\mathrm{j})$ as the corresponding systematic errors are generated in small magnitude in the regions (box 1 of 2003 and 2004 in figure 4) shown in figure 1 .

The positive and negative values of the flux term are assumed to be the convergence and divergence respectively in order to match the physical meaning of triad interaction with that of the convergence and divergence. Wavenumber spectra of the systematic error flux are computed following the methodology given in section 2.2 and are appeared in figures 5, 6 and 7 for the year 2001, 2003 and 2004 , respectively. There is no peak observed at the initial forecast period up to 2 days during 2001 in figure 5. Subsequently, the error energy converges to wavenumber 1 . It has also been observed that the error diverges from wavenumber 4 during 5-7 day forecasts (figure 5). Similarly the error flux of the year of 2003 shows a small peak associated with wavenumber 4 in 3 and 4-day forecasts (figure 6). Gradually, the peak is appeared to be stronger and shifted to the largest scale. Similar type of results are found during 2004 (figure 7) with the initial convergent mode of error at wavenumber 6 and finally, the error at the ultra-long scales of wavenumbers 1 and 4 . Figure 8 depicts the spectral representation of the nonlinear conversion term in error growth during 2003. The spectra delineate that the dominant mode is associated with wavenumber 4 at 4-day forecast and the mode is becoming stronger with the increase of lead time. It may also be observed from figure 8 that the wavenumber band $10-14$ is losing error energy in 6-8 day forecasts. The common feature obtained from the spectral analysis of the inertial terms is that there is hardly a strong positive peak observed at the initial forecast period up to 2 days. Gradually, a peak appears at the planetary scales and is getting stronger with the increase of the forecast period. An estimation of the energy 


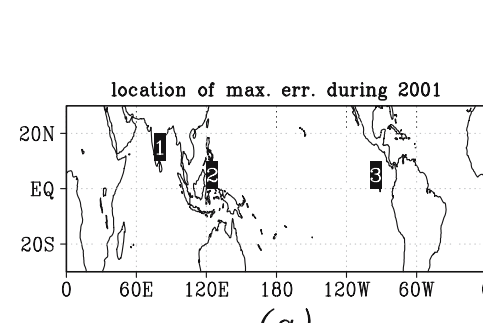

(a)
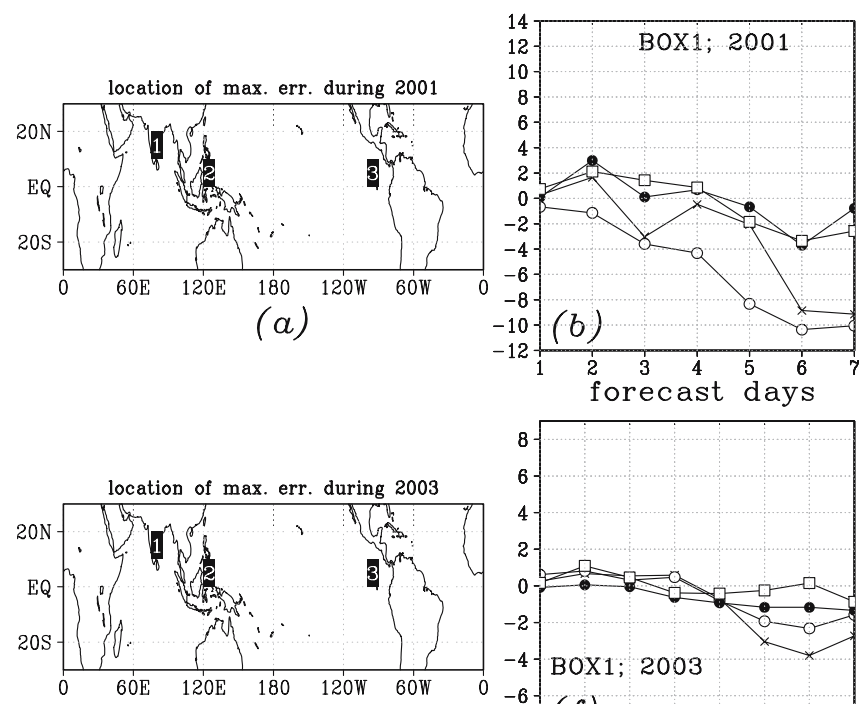

(e)

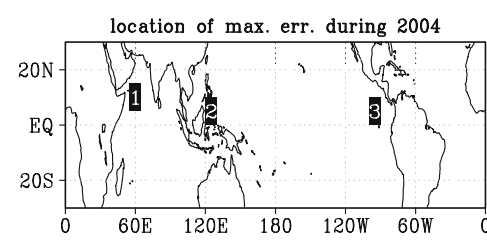

(i)

RESIDUAL
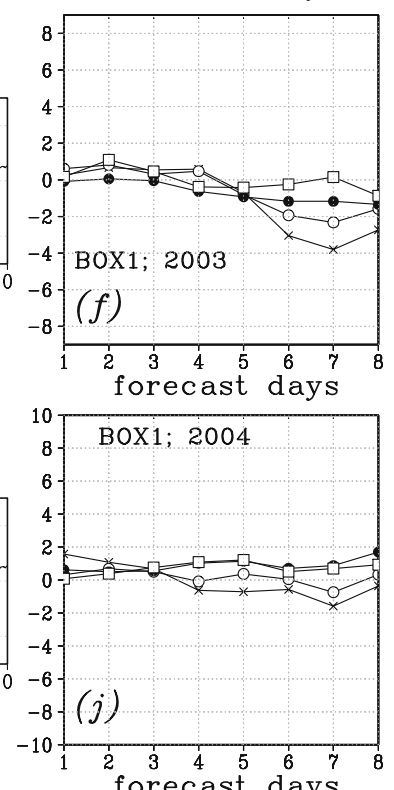

FLUX
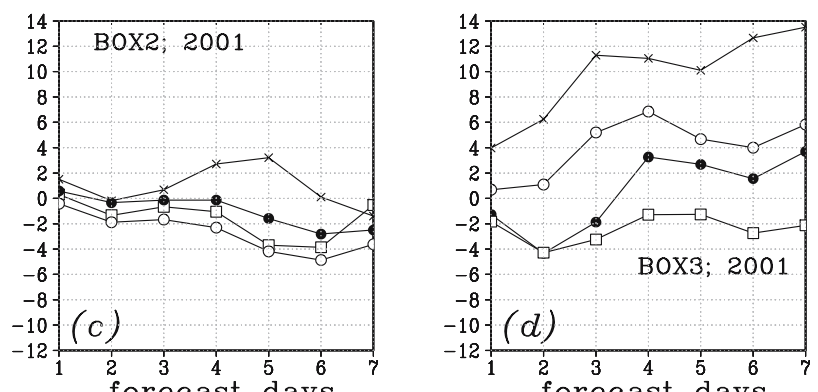
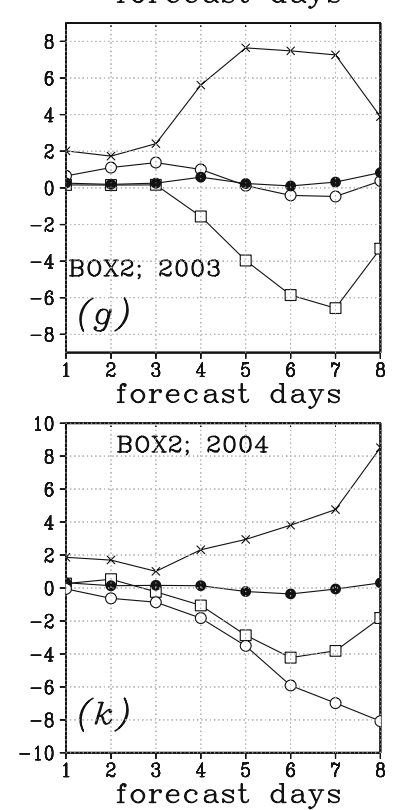

GENERATION
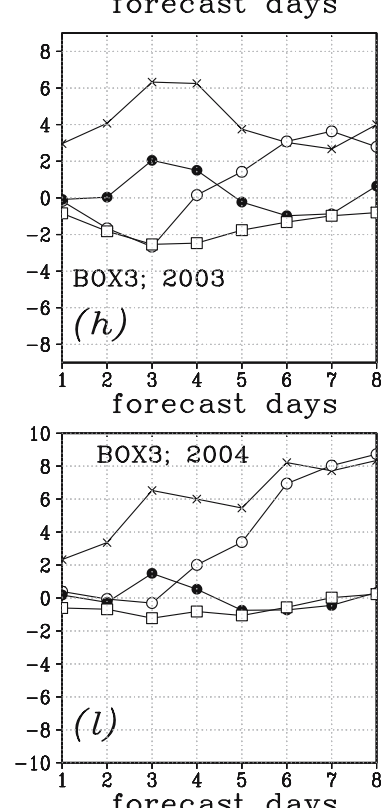

forecast days CONVERSION

Figure 4. First column (a, e, i) shows the location of maximum systematic error denoted by $10^{\circ} \times 10^{\circ}$ box marked with 1,2 and 3 at different years. The line graph of the right hand side terms of the error growth rate equations averaged over different boxes in different years are shown with the increase of the forecast days in figures $(b, c, d, f, g, h, j, k, l)$. The corresponding box and year are shown in each line graph diagram.

budget of the ultra-long waves (wavenumbers 1-4) by Gall et al (1979) revealed that the initial kinetic energy source of the planetary waves is the conversion of the eddy available potential energy to the eddy kinetic energy but not the nonlinear transfer between various wavenumbers. It can be inferred from Gall et al's (1979) findings that the convective process might be important for the initial kinetic energy of the planetary scale waves. In addition to this, Tribbia and Baumhefner (2004) in their perfect model twin experiment showed that 1-day forecast error was in situ or advected growth of the existing scale not the scale interactions but by 3-day forecasts, nonlinearity was prominent and considerable scale interactions had taken place. Now the typical questions would be: Does the error propagate from wavenumbers 4 to 1 at 5-7 day forecasts in the year 2001 (figure 5)? How the peak convergent mode has been shifted from wavenumber 6 to the planetary scales in figure 7 ? In the spectra of the conversion term (figure 8), is the error energy transported from the small scale waves of wavenumber band 10-14 to the ultralong scale of wavenumber 4 and responsible for making the error in planetary scale stronger with the increase of the forecast time? What is the mechanism of the non-linear upscale error transfer? According to Tribbia and Baumhefner's (2004) result has the scale interaction taken a significant role for cascading the error from small to the planetary scales and made the ultra-long scale difficult to forecast in the course of prediction? The wave-wave exchange of error energy through individual triads may possibly be the only mathematical device to visualize the scale interaction and to answer the above queries. It is not possible to exhibit the triad interactions for all the wavenumbers. The consistently dominant modes are chosen based on figures 5-8. These are wavenumber 1 of error flux during 2001, 2003 and 2004 and the wavenumber 4 of conversion term in 2003 as there is a consistent gain of error energy in these scales. 

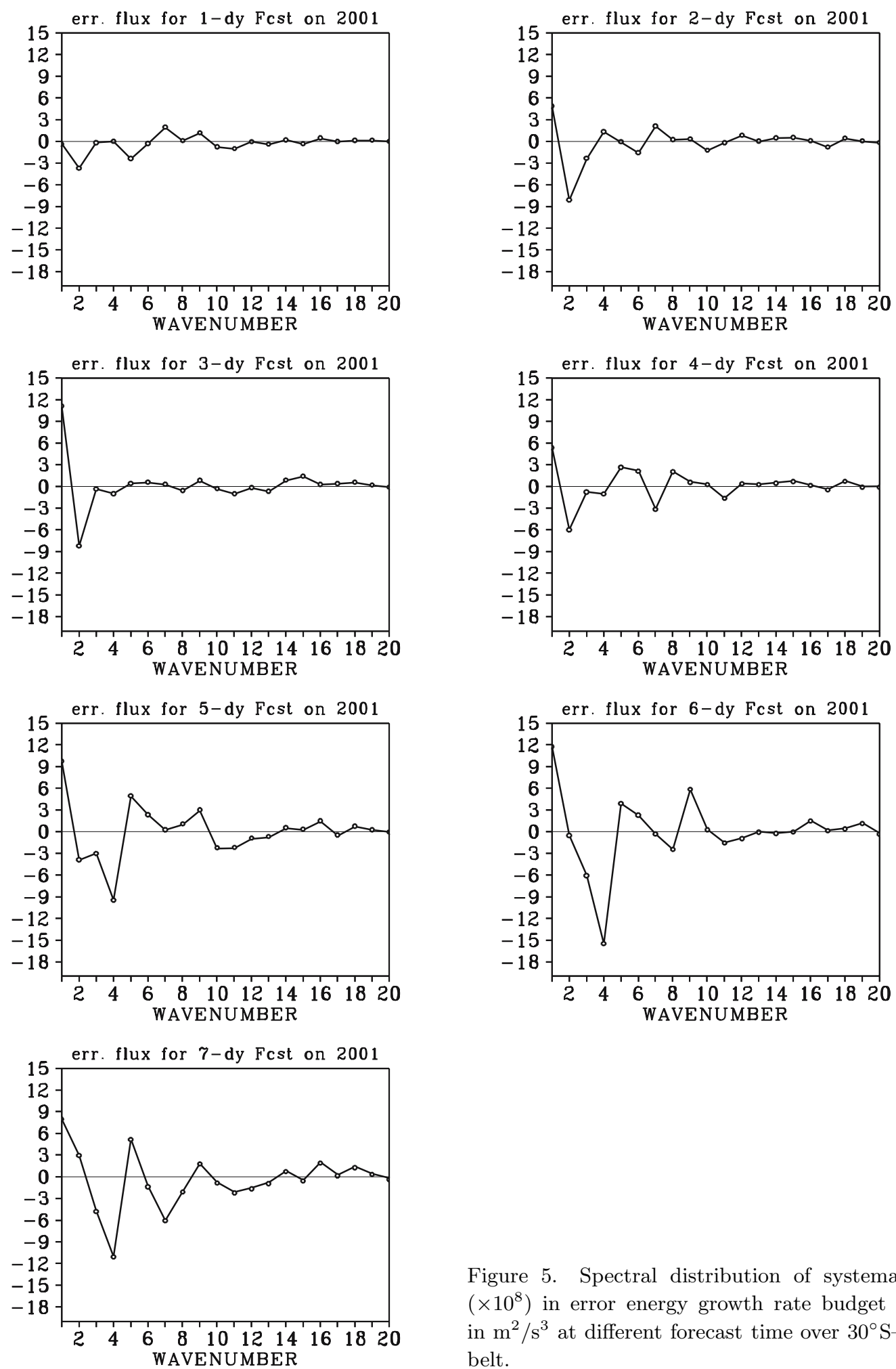

Figure 5. Spectral distribution of systematic error flux $\left(\times 10^{8}\right)$ in error energy growth rate budget for JJA, 2001 in $\mathrm{m}^{2} / \mathrm{s}^{3}$ at different forecast time over $30^{\circ} \mathrm{S}-30^{\circ} \mathrm{N}$ tropical belt.

Wavenumber 11 of the error energy conversion during 2003 that loses energy increasingly during 5 to 8-day forecasts (figure 8) is also considered. Next paragraph will describe the mechanism of upscale error transfer through wave-wave exchange of error energy in triad interactions.

In a triad interaction $(k, m, n)$ three waves of wavenumber $k, m$ and $n$ while interacting among themselves the wave $(k)$ either receives or loses energy to the other two waves $m$ and $n$ according to the positive or negative value of the triad interaction, respectively satisfying the 

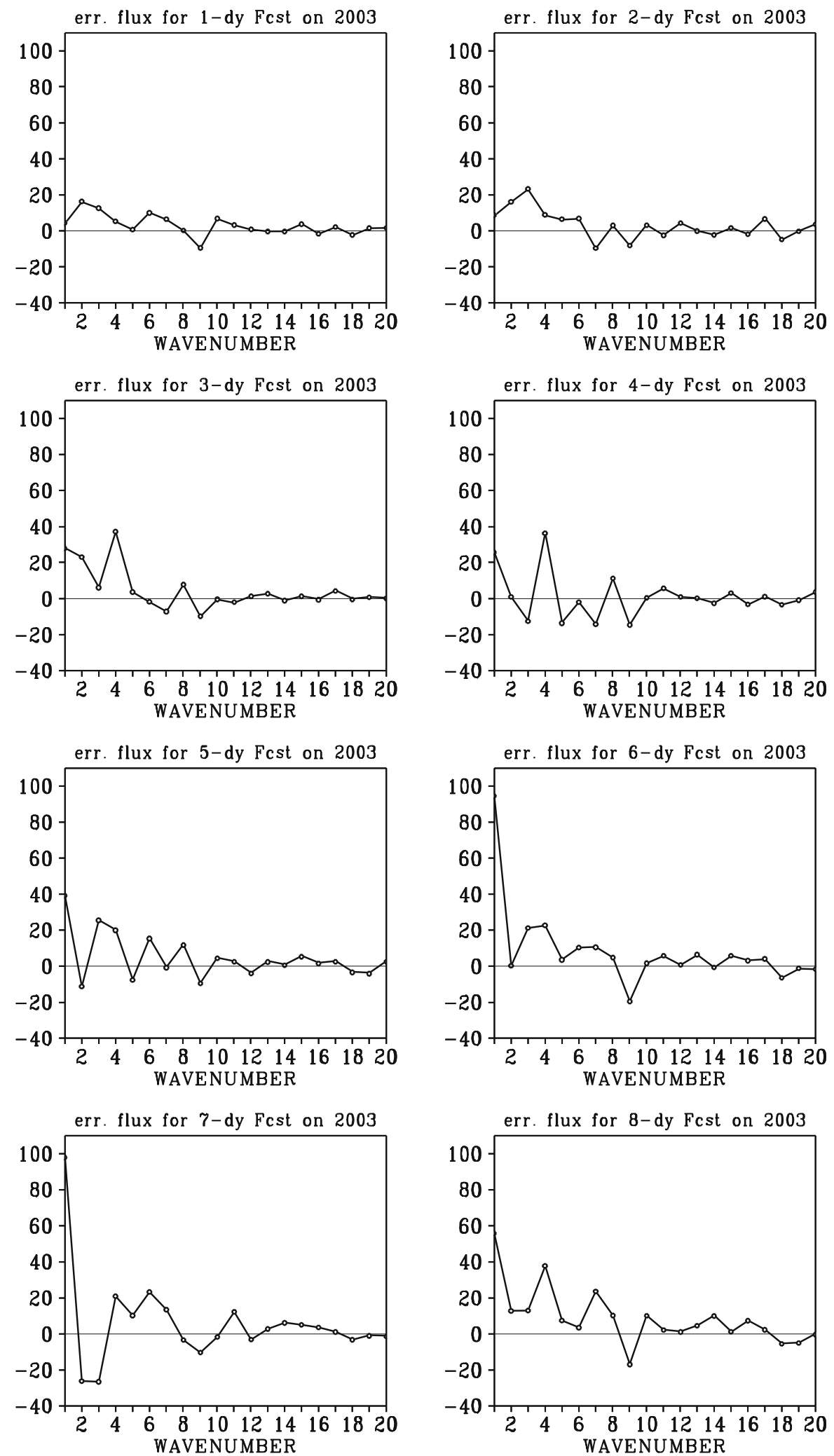

Figure 6. Same as figure 5, but for JJAS 2003 during 1-8 day forecasts.

selection rule $k=m+n$ or $k=|m-n|$. Therefore, consistently dominant positive or negative peaks in the spectral energy of any wave $(k)$ shown in figures 5-8 are associated with the larger and smaller number as well as magnitude of the positive value triads in comparison to the number and magnitude of the negative value triads, respectively after combining all the possible triads 



Figure 7. Same as figure 6, but for JJAS 2004.

associated with the spectra of $k$ for $k=m-n$, $k=n-m$ and $k=m+n$. The triad interaction of systematic error flux is computed from the equation (4) shown in an Appendix. The same for the nonlinear conversion term is evaluated from the similar type expression like equation (4). The $\mathrm{x}$-axis shows the plethora of triad $k, m$ and $n$ separated by comma and the y-axis indicates the 



Figure 8. The spectra of systematic error conversion term $\left(\times 10^{8}\right)$ in error growth rate budget equation for JJAS 2003 in $\mathrm{m}^{2} / \mathrm{s}^{3}$ at $850 \mathrm{hPa}$ over $30^{\circ} \mathrm{S}-30^{\circ} \mathrm{N}$ tropical belt for different days of forecast.

measure of error flux or conversion attributed to each triad interaction in the figures of triad spectra. A bunch of triads or a single triad responsible for the net gain of error energy of any wavenumber $k$ interacting with the two waves of wavenumber $m$ and $n$ are symbolized as ' $\longleftrightarrow$ ' whereas the net loss of error is shown by the symbol '— $\longleftarrow$ ' and neither loss nor gain of energy is 


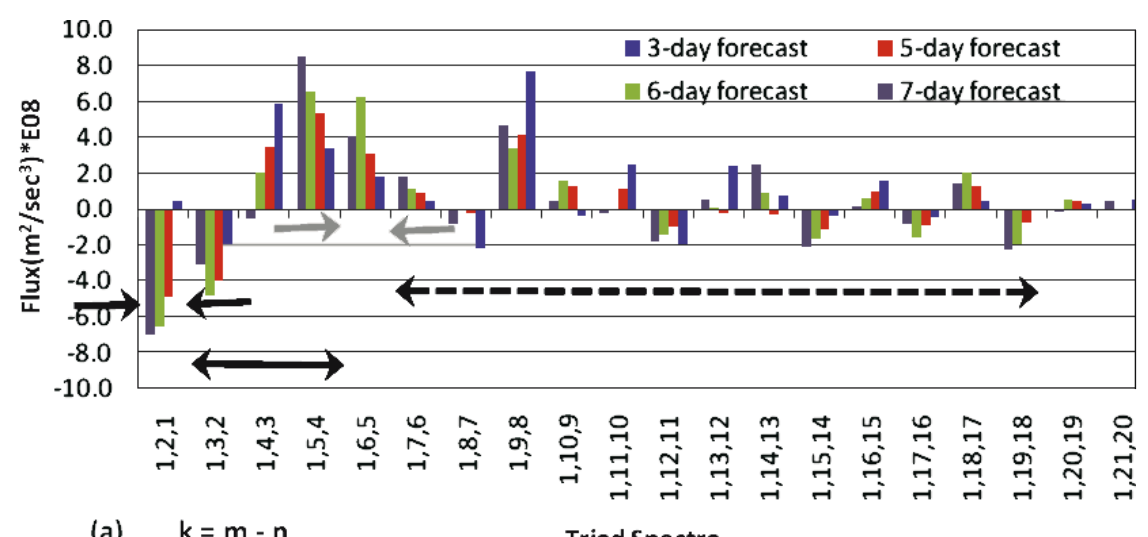

(a) $\quad k=m-n$

Triad Spectra

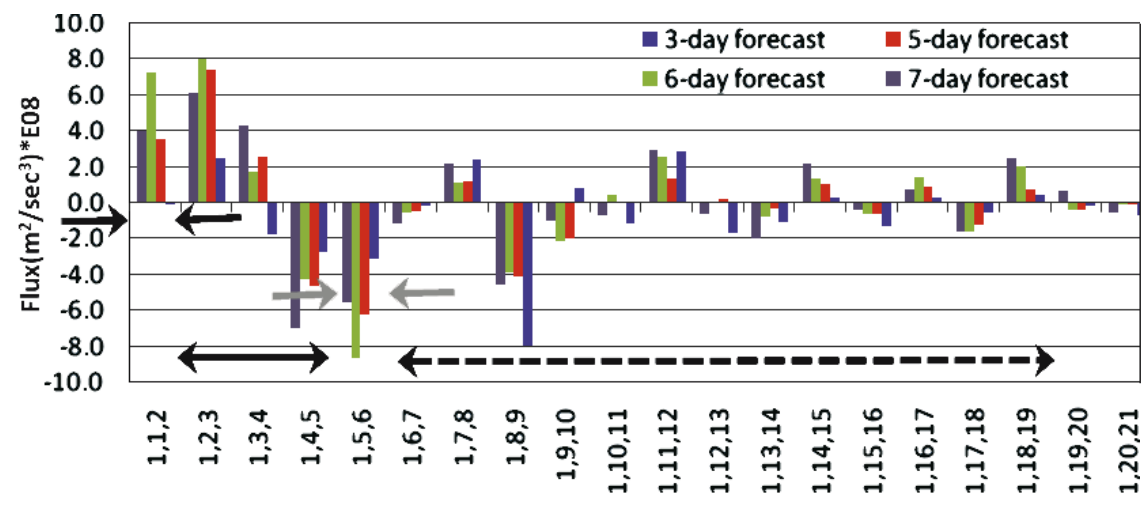

(b) $k=n-m$

Triad Spectra

Figure 9. Individual triad interaction spectra of the systematic error flux of error energy equation at wavenumber $k=1$ for (a) $k=m-n$ and (b) $k=n-m$ on 3,5,6 and 7-day forecasts during 2001. The sign ' $\longleftrightarrow$ ' indicates the triads through which wavenumber $k$ is gaining energy. The wavenumber $k$ is losing energy in terms of the triads shown by '— $\longleftarrow$ '. Triads are almost equal and opposite marked by ' $\leftarrow---\rightarrow$ '.

denoted by ' $\leftarrow---\rightarrow$ ' in all the spectra for $k=m-n$, $k=n-m$ and $k=m+n$. First twenty triad interactions are shown in all spectra as the interactions are insignificant after twenty triads. The figure $9(\mathrm{a}$ and $\mathrm{b})$ elucidates the triad spectra associated with wavenumber 1 for $k=m-n$ and $k=n-m$ respectively of the systematic error flux in 2001. Addition of the two spectra has shown the larger positive value triads in comparison to the negative value triads at the wavenumber band $2-5$ during the $3,5,6$ and 7-day forecast period. The implication is that there is a net transfer of error energy from the above wavenumber band to the ultra-long wave of wavenumber 1 through the respective triads $(1,3,2) ;(1,4,3)$ and $(1,5,4)$ and $(1,2,3) ;(1,3,4)$ and $(1,4,5)$ of spectra for $k=m-n$ and $k=n-m$ respectively indicating by the symbol ' $\longleftrightarrow$ '. The only triplet interactions through which wavenumber 1 loses small amount of error energy are those with wavenumbers $(1,2)$ denoted by ' $\longleftrightarrow \longleftarrow$ ' and those with wavenumbers $(5,6)$ denoted by ' $\longrightarrow \longleftarrow$ '. Other triads are almost negated as they are nearly equal and opposite in the two spectra marked in figure as
' $r---\rightarrow$ '. The reason for strong divergence of error associated with wavenumber 4 shown in figure 5 may be explained as the nonlinear spreading of systematic error energy from wavenumbers 4 to 1 in terms of the respective triads $(1,4,3) ;(1,5,4)$ and $(1,3,4)$ and $(1,4,5)$ observed clearly in figure 9. Triad interaction analysis of wavenumber 1 in the year 2003 depicts that the wavenumber 1 is gaining the energy from the larger wavenumbers $2-4$ and from wavenumbers 5 and 6 in terms of their respective triads shown by ' $\longleftrightarrow$ ' and ' $\longleftrightarrow$ ', respectively in figure 10 ( $a$ and $b$ ). Rest of the triads is nullified after adding both spectra marked by ' $r---\rightarrow$ ' as they are equal and opposite in nature. Similarly in the year 2004, the associated triads of the wavenumber range 2-6 and the pair of wavenumbers 8 and 9 denoted by the symbol ' $\longleftrightarrow$ ' and ' $\longleftrightarrow$ ' respectively loses error energy to the largest scale at 5-8 day forecasts implying the upscale propagation of error energy shown combining the spectra for $k=m-n$ (figure 11a) and $k=n-m$ (figure 11b). Small amount of error energy is transferred from wavenumber 1 to wavenumbers $(1,2)$ in the triads $(1,1,2)$ and $(1,2,1)$ in 


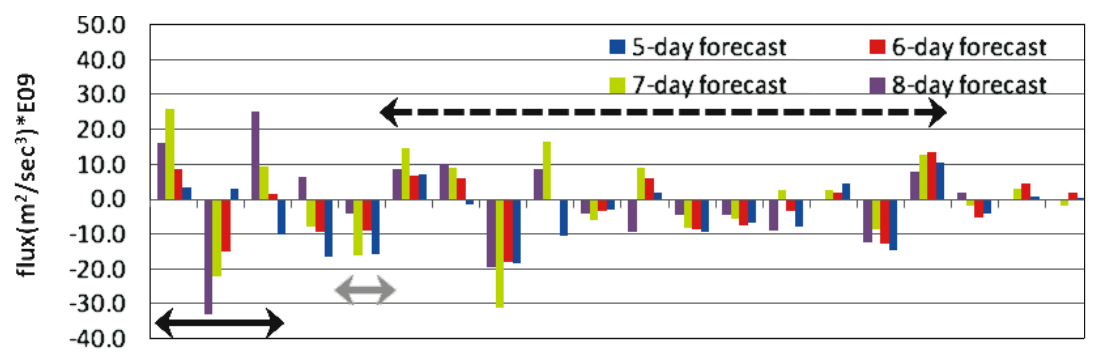



(a) $\mathrm{k}=\mathrm{m}-\mathrm{n} \quad$ Triad Spectra

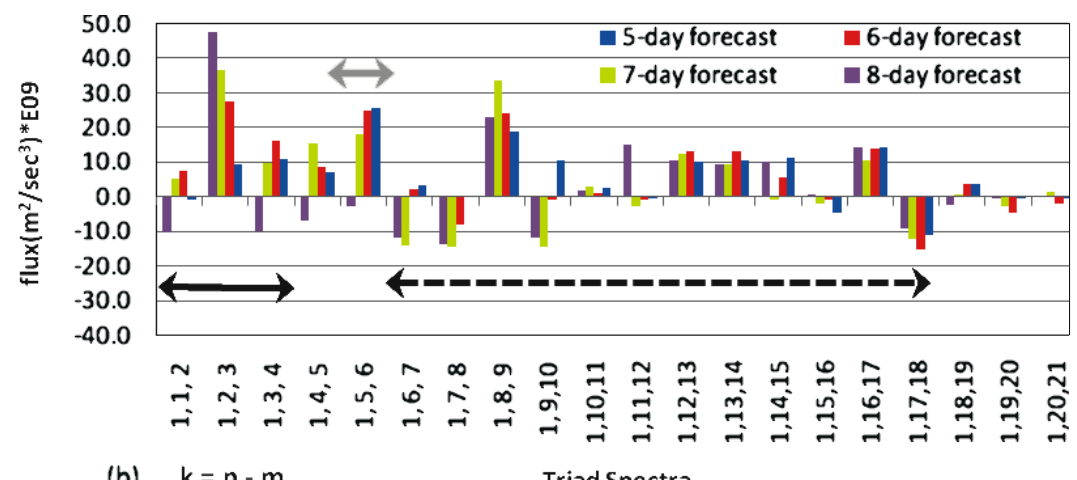

Figure 10. Same as figure 9, but for the year 2003 on 5, 6, 7 and 8-day forecasts.

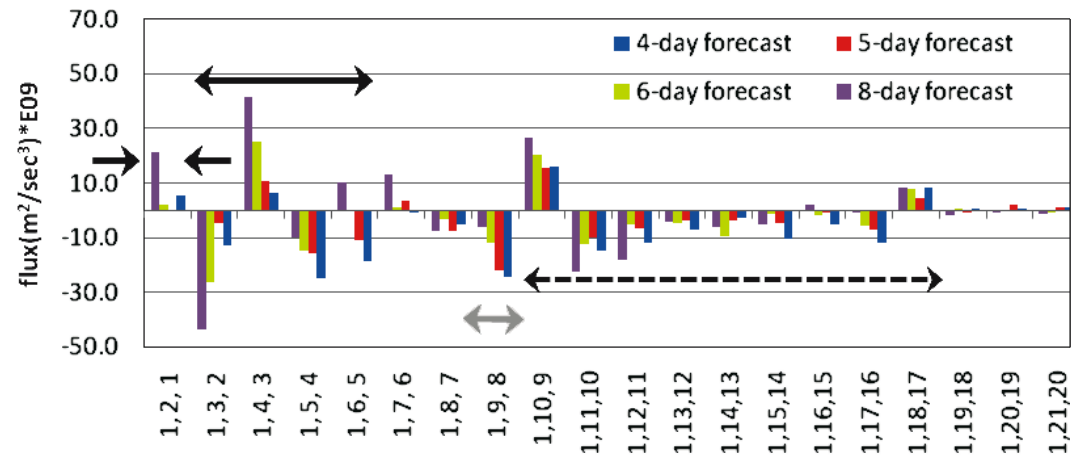

(a) $k=m-n$

Triad Spectra

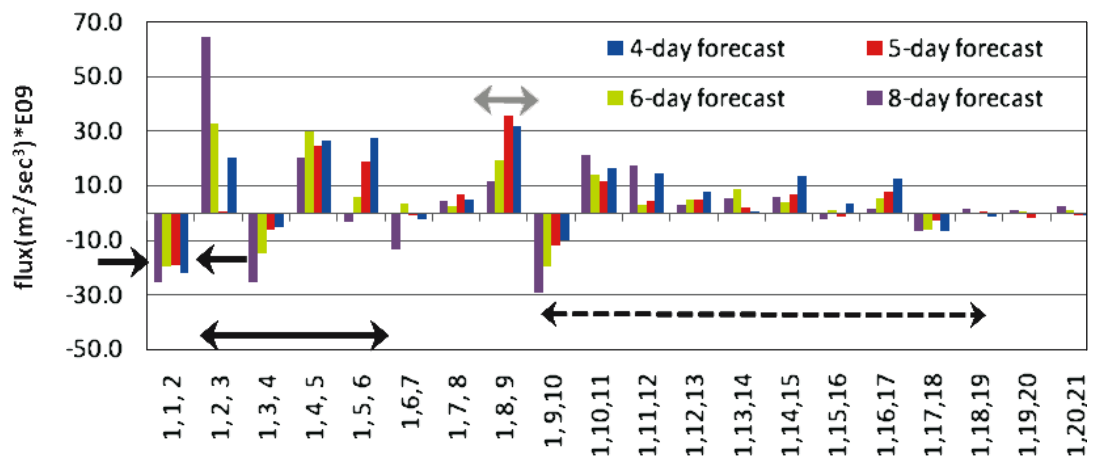

(b) $k=n-m$

Triad Spectra

Figure 11. Same as figure 9, but for 4-8 day forecasts in the year 2004 . 

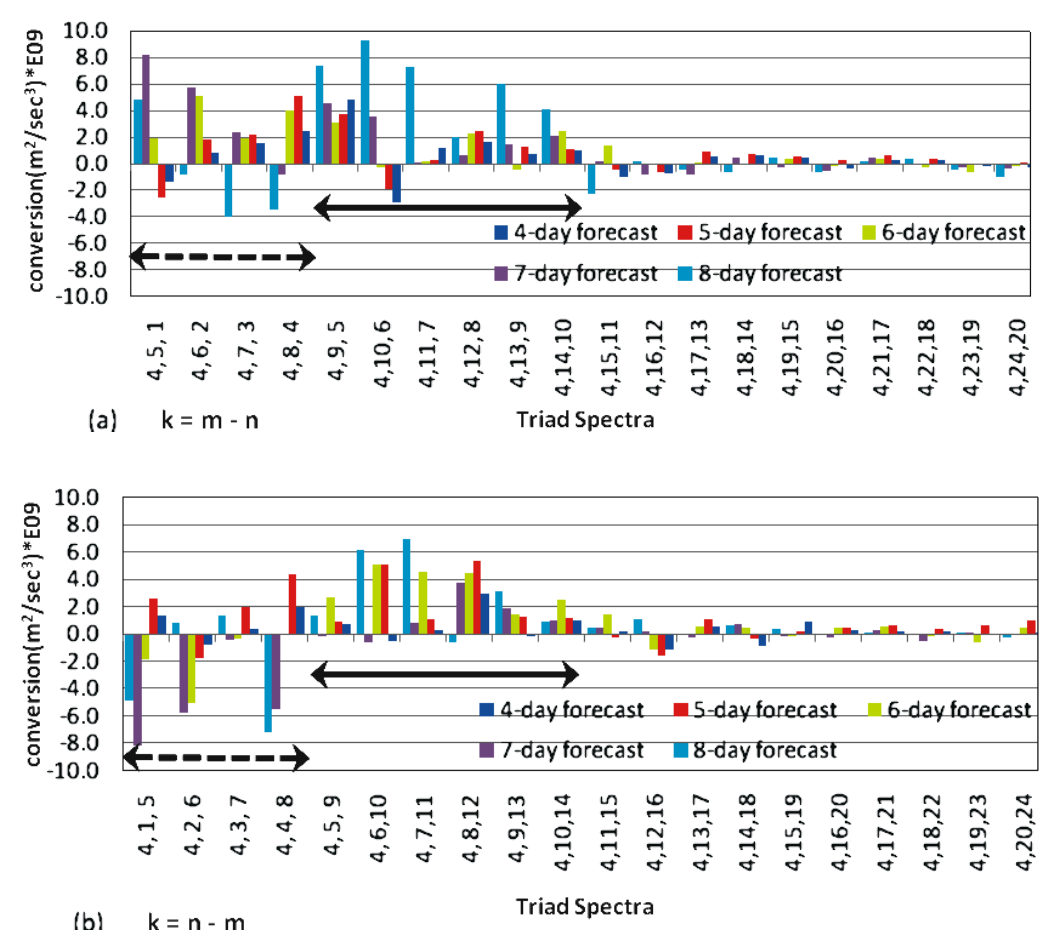

Figure 12. Individual triad interaction spectra of systematic error conversion term in error growth rate budget equation at wavenumber $k=4$ for (a) $k=m-n$ and (b) $k=n-m$ at $4-8$ day forecasts during 2003. The meaning of the arrows shown is same as in figure 9 .

figure $11(\mathrm{a}$ and $\mathrm{b})$ respectively shown by a symbol ' $\rightarrow \leftarrow$ '. Other triads denoted by ' $\leftarrow---\rightarrow$ ' are cancelled each other during 2004. There is a net gain of error energy of wavenumber 4 from the small scale waves of wavenumbers $5-14$ combining the spectra for $k=m-n$ (figure 12a) and $k=n-m$ (figure 12b) marked by a symbol ' $\longleftrightarrow$ '. Other triads in figure 12 associated with the higher scales compared to wavenumber 4 are almost equal and opposite shown by ' $r--\rightarrow$ ' and hence, they are neutralized. The number and magnitude of the negative value triads is more compared to that of the positive value triads in the spectral decomposition of wavenumber 11 during 2003 shown in figure 13. Wavenumber 11 loses error energy to wavenumbers 1 and 2 in the pair of triads $(11,1,12)$ and $(11,2,13)$ shown as ' $\rightarrow \leftarrow$ ' in the spectra for $k=n-m$ (figure 13b). The upscale spreading of error from wavenumber 11 to wavenumber range 2-9 is visualized in the spectra for $k=m+n$ (figure 13c) denoted by ' $\rightarrow \leftarrow$ ' and ' $\longleftarrow$ '. Only the wavenumber 5 and 6 have no contribution to wavenumber 11 as the corresponding triads are cancelled each other symbolized as ' $---\rightarrow$ '. Rests of the triads are insignificant. It is inferred from the triad spectra of wavenumber 11 that the negative mode of wavenumber 11 appeared after 4-day forecasts in figure 8 is due to the transport of the error energy from wavenumber 11 to the higher scale waves during 5-8 day forecasts observed in figure 13 .
It is concluded from the comprehensive analysis that the non-linear error propagation from the small to large scale through the scale interaction is the significant inherent dynamical process evolved within the prediction system after 3-day forecast that is in agreement with the results obtained by Tribbia and Baumhefner (2004). The mechanism of non-linear upscale transfer of error is the wavewave exchange of energy among the participating triads through which planetary scale error become dominant with the increase of forecast lead after the initiation of error attributed to the physical process described in section 4.1.

\section{Conclusions}

The large build-up of error in the planetary waves is inevitable in medium range weather forecast of the numerical weather prediction model. It not only reduces the skill of the medium range forecast but acts also as a barrier in the dynamical extended range prediction. So, before devising a methodology to improve the forecast it is essential to unravel the exact theory that causes the poor predictability of lower tropospheric ultralong waves in tropics. First time, as an initial attempt in this direction, GFS model analysisforecast system is utilized extensively at $850 \mathrm{hPa}$ level over $30^{\circ} \mathrm{S}-30^{\circ} \mathrm{N}$ tropical belt. 

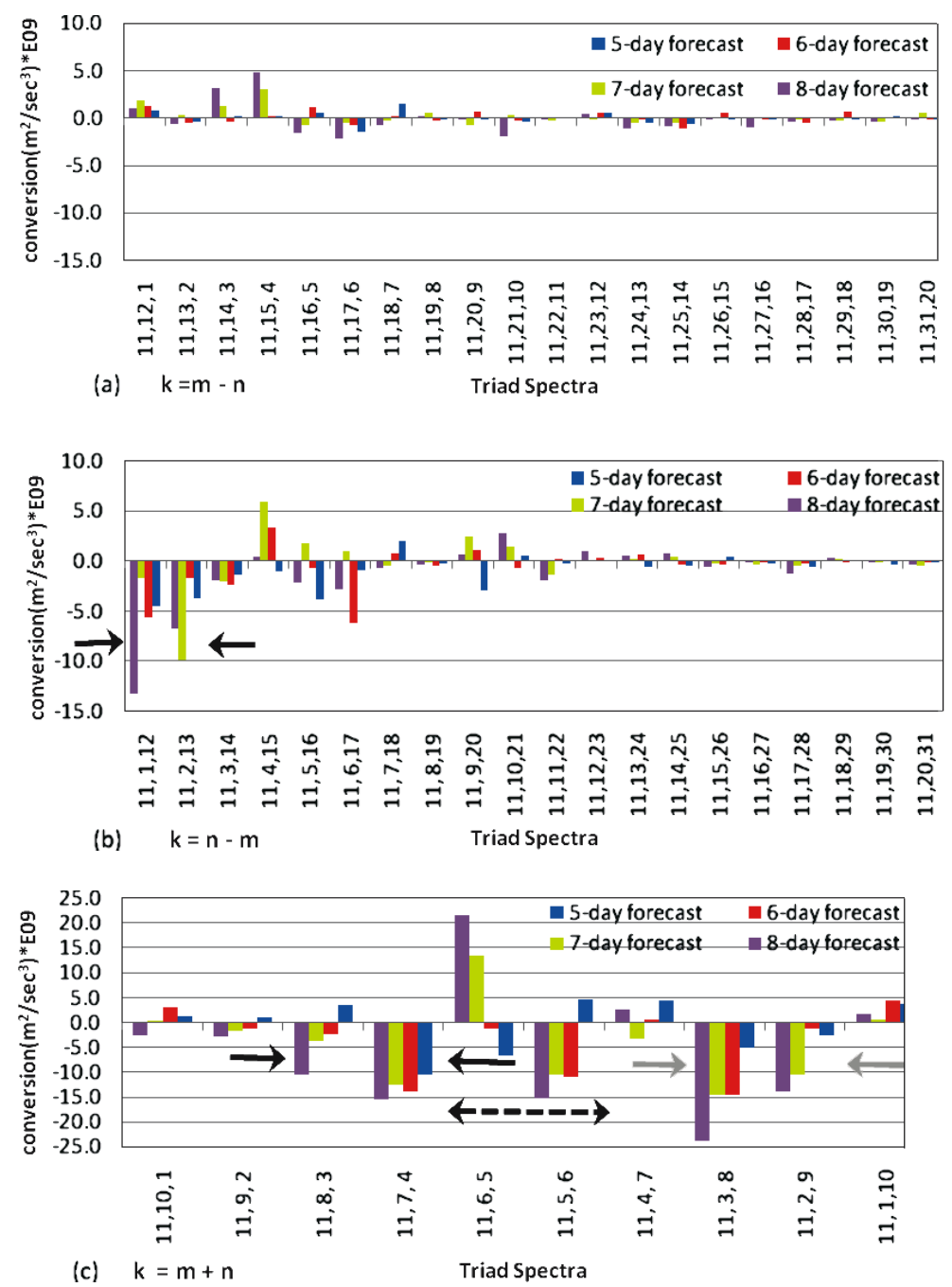

Figure 13. Same as figure 12, but for the triad spectra at wavenumber $k=11$ on $5-8$ day forecasts during 2003 .

An exhaustive analysis of the GFS model has shown that the systematic error is generated initially due to the inadequate representation of different physical processes such as the formulation of cumulus clouds applied in the model. At the subsequent realization of forecasts, there is a non-linear upscale spreading of error through multiple scale interactions (computed from the wave-wave exchange of error energy) that builds error systematically in the planetary scales. This successive development of ultra-long scale error amplifies the uncertainty that acts as a limiting factor to predict accurately the lower tropospheric ultra-long waves in the tropical region during medium range forecasts.

In a further deeper insight into the evolution of error it may be conjectured that the systematic error may perhaps generate initially from the inaccuracy to parameterize the sub-grid scale processes like cumulus convection, boundary layer process, etc., in the spectrally unresolved region and subsequently appears in the lowest resolvable scales through the process of triad interaction that eventually spreads to the ultra-long scale. This may be described in the following hypothetical schematic diagram.

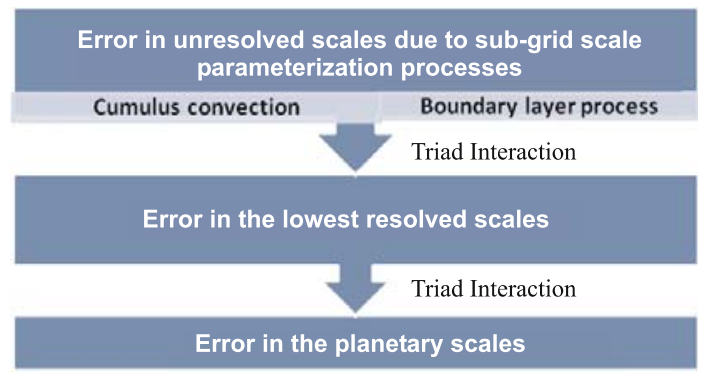

The objective of this paper has been explained using one model only. To generalize the findings and to make it robust, thorough investigations utilizing different models are necessary with an application of the above results in a broader term like the predictability of intra-seasonal oscillations fitting with the qualitative conclusions. It would have significant implications in the numerical 
weather prediction to investigate on the following aspects for the improvement of ultra-long scale predictability.

- To propose a methodology of preventing the upscale propagation of error within the prediction system so that the initial error will not mingle with the error in planetary scales.

- To improve the forecast of the small scale systems, this can ascertain the reduction of uncertainty in the ultra-long scales over tropics.

\section{Acknowledgements}

The author is grateful to the Director, IITM for improving the manuscript and for constant encouragement and to the Head, TS division for help and support. He is also thankful to NCEP for making GFS model output freely available through the GFS site. Thanks are due to Brian Doty, COLA for GrADS software. The author thanks the anonymous referees for their constructive comments.

\section{Appendix}

$\sum_{k} F^{s}(t)$

$$
\begin{aligned}
& =-\frac{1}{4}\left(\sum_{k=m+n}+\sum_{k=m-n}+\sum_{k=n-m}\right)\left\{\begin{array}{c}
u_{f} C_{k} \cdot u_{e}^{s} C_{m}\left(\frac{\partial u_{e}^{s}}{\partial x}\right) C_{n}+u_{f} C_{k} \cdot v_{e}^{s} C_{m} \cdot\left(\frac{\partial v_{e}^{s}}{\partial x}\right) C_{n} \\
+\frac{1}{2}\left(\frac{\partial u_{f}}{\partial x}\right) C_{k} \cdot u_{e}^{s} C_{m} \cdot u_{e}^{s} C_{n}+\frac{1}{2}\left(\frac{\partial u_{f}}{\partial x}\right) C_{k} \cdot v_{e}^{s} C_{m} \cdot v_{e}^{s} C_{n} \\
+v_{f} C_{k} \cdot u_{e}^{s} C_{m} \cdot\left(\frac{\partial u_{e}^{s}}{\partial y}\right) C_{n}+v_{f} C_{k} \cdot v_{e}^{s} C_{m} \cdot\left(\frac{\partial v_{e}^{s}}{\partial y}\right) C_{n} \\
+\frac{1}{2}\left(\frac{\partial v_{f}}{\partial y}\right) C_{k} \cdot u_{e}^{s} C_{m} \cdot u_{e}^{s} C_{n}+\frac{1}{2}\left(\frac{\partial v_{f}}{\partial y}\right) S_{k} \cdot v_{e}^{s} C_{m} \cdot v_{e}^{s} C_{n} \\
+\left(\frac{\partial u_{f}}{\partial x}\right) C_{k} \cdot u_{e}^{s} C_{m} \cdot u_{e}^{r} C_{n}+u_{f} C_{k} \cdot\left(\frac{\partial u_{e}^{s}}{\partial x}\right) C_{m} \cdot u_{e}^{r} C_{n} \\
+u_{f} C_{k} \cdot u_{e}^{s} C_{m} \cdot\left(\frac{\partial u_{e}^{r}}{\partial x}\right) C_{n}+\left(\frac{\partial u_{f}}{\partial x}\right) C_{k} \cdot v_{e}^{s} C_{m} \cdot v_{e}^{r} C_{n} \\
+u_{f} C_{k} \cdot v_{e}^{s} C_{m} \cdot\left(\frac{\partial v_{e}^{r}}{\partial x}\right) C_{n}+\left(\frac{\partial v_{f}}{\partial y}\right) C_{k} \cdot u_{e}^{s} C_{m} \cdot u_{e}^{r} C_{n} \\
+v_{f} C_{k} \cdot\left(\frac{\partial u_{e}^{s}}{\partial y}\right) C_{m} \cdot u_{e}^{r} C_{n}+u_{f} C_{k} \cdot\left(\frac{\partial v_{e}^{s}}{\partial x}\right) C_{m} \cdot v_{e}^{r} C_{n} \\
+v_{f} C_{k} \cdot u_{e}^{s} C_{m} \cdot\left(\frac{\partial u_{e}^{r}}{\partial y}\right) C_{n}+\left(\frac{\partial v_{f}}{\partial y}\right) C_{k} \cdot v_{e}^{s} C_{m} \cdot v_{e}^{r} C_{n} \\
+v_{f} C_{k} \cdot\left(\frac{\partial v_{e}^{s}}{\partial y}\right) C_{m} \cdot v_{e}^{r} C_{n}+v_{f} C_{k} \cdot v_{e}^{s} C_{m} \cdot\left(\frac{\partial v_{e}^{r}}{\partial y}\right) C_{n}
\end{array}\right\} \\
& -\frac{1}{4}\left(-\sum_{k=m+n}+\sum_{k=m-n}+\sum_{k=n-m}\right) \\
& \left\{\begin{array}{c}
u_{f} C_{k} \cdot u_{e}^{s} S_{m}\left(\frac{\partial u_{e}^{s}}{\partial x}\right) S_{n}+u_{f} C_{k} \cdot v_{e}^{s} S_{m} \cdot\left(\frac{\partial v_{e}^{s}}{\partial x}\right) S_{n} \\
+\frac{1}{2}\left(\frac{\partial u_{f}}{\partial x}\right) C_{k} \cdot u_{e}^{s} S_{m} \cdot u_{e}^{s} S_{n}+\frac{1}{2}\left(\frac{\partial u_{f}}{\partial x}\right) C_{k} \cdot v_{e}^{s} S_{m} \cdot v_{e}^{s} S_{n} \\
\quad+v_{f} C_{k} \cdot u_{e}^{s} S_{m} \cdot\left(\frac{\partial u_{e}^{s}}{\partial y}\right) S_{n}+v_{f} C_{k} \cdot v_{e}^{s} S_{m} \cdot\left(\frac{\partial v_{e}^{s}}{\partial y}\right) S_{n} \\
\quad+\frac{1}{2}\left(\frac{\partial v_{f}}{\partial y}\right) C_{k} \cdot u_{e}^{s} S_{m} \cdot u_{e}^{s} S_{n}+\frac{1}{2}\left(\frac{\partial v_{f}}{\partial y}\right) C_{k} \cdot v_{e}^{s} S_{m} \cdot v_{e}^{s} S_{n} \\
\quad+\left(\frac{\partial u_{f}}{\partial x}\right) C_{k} \cdot u_{e}^{s} S_{m} \cdot u_{e}^{r} S_{n}+u_{f} C_{k} \cdot\left(\frac{\partial u_{e}^{s}}{\partial x}\right) S_{m} \cdot u_{e}^{r} S_{n} \\
\quad+u_{f} C_{k} \cdot u_{e}^{s} S_{m} \cdot\left(\frac{\partial u_{e}^{r}}{\partial x}\right) S_{n}+\left(\frac{\partial u_{f}}{\partial x}\right) C_{k} \cdot v_{e}^{s} S_{m} \cdot v_{e}^{r} S_{n} \\
\quad+u_{f} C_{k} \cdot v_{e}^{s} S_{m} \cdot\left(\frac{\partial v_{e}^{r}}{\partial x}\right) S_{n}+\left(\frac{\partial v_{f}}{\partial y}\right) C_{k} \cdot u_{e}^{s} S_{m} \cdot u_{e}^{r} S_{n} \\
\quad+v_{f} C_{k} \cdot\left(\frac{\partial u_{e}^{s}}{\partial y}\right) S_{m} \cdot u_{e}^{r} S_{n}+u_{f} C_{k} \cdot\left(\frac{\partial v_{e}^{s}}{\partial x}\right) S_{m} \cdot v_{e}^{r} S_{n} \\
\quad+v_{f} C_{k} \cdot u_{e}^{s} S_{m} \cdot\left(\frac{\partial u_{e}^{r}}{\partial y}\right) S_{n}+\left(\frac{\partial v_{f}}{\partial y}\right) C_{k} \cdot v_{e}^{s} S_{m} \cdot v_{e}^{r} S_{n} \\
\quad+v_{f} C_{k} \cdot\left(\frac{\partial v_{e}^{s}}{\partial y}\right) S_{m} \cdot v_{e}^{r} S_{n}+v_{f} C_{k} \cdot v_{e}^{s} S_{m} \cdot\left(\frac{\partial v_{e}^{r}}{\partial y}\right) S_{n}
\end{array}\right\}
\end{aligned}
$$




$$
\begin{aligned}
& -\frac{1}{4}\left(\sum_{k=m+n}-\sum_{k=m-n}+\sum_{k=n-m}\right)\left\{\begin{array}{r}
u_{f} S_{k} \cdot u_{e}^{s} C_{m}\left(\frac{\partial u_{e}^{s}}{\partial x}\right) S_{n}+u_{f} S_{k} \cdot v_{e}^{s} C_{m} \cdot\left(\frac{\partial v_{e}^{s}}{\partial x}\right) S_{n} \\
+\frac{1}{2}\left(\frac{\partial u_{f}}{\partial x}\right) S_{k} \cdot u_{e}^{s} C_{m} \cdot u_{e}^{s} S_{n}+\frac{1}{2}\left(\frac{\partial u_{f}}{\partial x}\right) S_{k} \cdot v_{e}^{s} C_{m} \cdot v_{e}^{s} S_{n} \\
+v_{f} S_{k} \cdot u_{e}^{s} C_{m} \cdot\left(\frac{\partial u_{e}^{s}}{\partial y}\right) S_{n}+v_{f} S_{k} \cdot v_{e}^{s} C_{m} \cdot\left(\frac{\partial v_{e}^{s}}{\partial y}\right) S_{n} \\
+\frac{1}{2}\left(\frac{\partial v_{f}}{\partial y}\right) S_{k} \cdot u_{e}^{s} C_{m} \cdot u_{e}^{s} S_{n}+\frac{1}{2}\left(\frac{\partial v_{f}}{\partial y}\right) S_{k} \cdot v_{e}^{s} C_{m} \cdot v_{e}^{s} S_{n} \\
+\left(\frac{\partial u_{f}}{\partial x}\right) S_{k} \cdot u_{e}^{s} C_{m} \cdot u_{e}^{r} S_{n}+u_{f} S_{k} \cdot\left(\frac{\partial u_{e}^{s}}{\partial x}\right) C_{m} \cdot u_{e}^{r} S_{n} \\
+u_{f} S_{k} \cdot u_{e}^{s} C_{m} \cdot\left(\frac{\partial u_{e}^{r}}{\partial x}\right) S_{n}+\left(\frac{\partial u_{f}}{\partial x}\right) S_{k} \cdot v_{e}^{s} C_{m} \cdot v_{e}^{r} S_{n} \\
+u_{f} S_{k} \cdot v_{e}^{s} C_{m} \cdot\left(\frac{\partial v_{e}^{r}}{\partial x}\right) S_{n}+\left(\frac{\partial v_{f}}{\partial y}\right) S_{k} \cdot u_{e}^{s} C_{m} \cdot u_{e}^{r} S_{n} \\
+v_{f} S_{k} \cdot\left(\frac{\partial u_{e}^{s}}{\partial y}\right) C_{m} \cdot u_{e}^{r} S_{n}+u_{f} S_{k} \cdot\left(\frac{\partial v_{e}^{s}}{\partial x}\right) C_{m} \cdot v_{e}^{r} S_{n} \\
+v_{f} S_{k} \cdot u_{e}^{s} C_{m} \cdot\left(\frac{\partial u_{e}^{r}}{\partial y}\right) S_{n}+\left(\frac{\partial v_{f}}{\partial y}\right) S_{k} \cdot v_{e}^{s} C_{m} \cdot v_{e}^{r} S_{n} \\
+v_{f} S_{k} \cdot\left(\frac{\partial v_{e}^{s}}{\partial y}\right) C_{m} \cdot v_{e}^{r} S_{n}+v_{f} S_{k} \cdot v_{e}^{s} C_{m} \cdot\left(\frac{\partial v_{e}^{r}}{\partial y}\right) S_{n}
\end{array}\right\} \\
& -\frac{1}{4}\left(\sum_{k=m+n}+\sum_{k=m-n}-\sum_{k=n-m}\right)\left\{\begin{array}{c}
u_{f} S_{k} \cdot u_{e}^{s} S_{m}\left(\frac{\partial u_{e}^{s}}{\partial x}\right) C_{n}+u_{f} S_{k} \cdot v_{e}^{s} S_{m} \cdot\left(\frac{\partial v_{e}^{s}}{\partial x}\right) C_{n} \\
+\frac{1}{2}\left(\frac{\partial u_{f}}{\partial x}\right) S_{k} \cdot u_{e}^{s} S_{m} \cdot u_{e}^{s} C_{n}+\frac{1}{2}\left(\frac{\partial u_{f}}{\partial x}\right) S_{k} \cdot v_{e}^{s} S_{m} \cdot v_{e}^{s} C_{n} \\
+v_{f} S_{k} \cdot u_{e}^{s} S_{m} \cdot\left(\frac{\partial u_{e}^{s}}{\partial y}\right) C_{n}+v_{f} S_{k} \cdot v_{e}^{s} S_{m} \cdot\left(\frac{\partial v_{e}^{s}}{\partial y}\right) C_{n} \\
+\frac{1}{2}\left(\frac{\partial v_{f}}{\partial y}\right) S_{k} \cdot u_{e}^{s} S_{m} \cdot u_{e}^{s} C_{n}+\frac{1}{2}\left(\frac{\partial v_{f}}{\partial y}\right) S_{k} \cdot v_{e}^{s} S_{m} \cdot v_{e}^{s} C_{n} \\
+\left(\frac{\partial u_{f}}{\partial x}\right) S_{k} \cdot u_{e}^{s} S_{m} \cdot u_{e}^{r} C_{n}+u_{f} S_{k} \cdot\left(\frac{\partial u_{e}^{s}}{\partial x}\right) S_{m} \cdot u_{e}^{r} C_{n} \\
+u_{f} S_{k} \cdot u_{e}^{s} S_{m} \cdot\left(\frac{\partial u_{e}^{r}}{\partial x}\right) C_{n}+\left(\frac{\partial u_{f}}{\partial x}\right) S_{k} \cdot v_{e}^{s} S_{m} \cdot v_{e}^{r} C_{n} \\
+u_{f} S_{k} \cdot v_{e}^{s} S_{m} \cdot\left(\frac{\partial v_{e}^{r}}{\partial x}\right) C_{n}+\left(\frac{\partial v_{f}}{\partial y}\right) S_{k} \cdot u_{e}^{s} S_{m} \cdot u_{e}^{r} C_{n} \\
+v_{f} S_{k} \cdot\left(\frac{\partial u_{e}^{s}}{\partial y}\right) S_{m} \cdot u_{e}^{r} C_{n}+u_{f} S_{k} \cdot\left(\frac{\partial v_{e}^{s}}{\partial x}\right) S_{m} \cdot v_{e}^{r} C_{n} \\
+v_{f} S_{k} \cdot u_{e}^{s} S_{m} \cdot\left(\frac{\partial u_{e}^{r}}{\partial y}\right) C_{n}+\left(\frac{\partial v_{f}}{\partial y}\right) S_{k} \cdot v_{e}^{s} S_{m} \cdot v_{e}^{r} C_{n} \\
+v_{f} S_{k} \cdot\left(\frac{\partial v_{e}^{s}}{\partial y}\right) S_{m} \cdot v_{e}^{r} C_{n}+v_{f} S_{k} \cdot v_{e}^{s} S_{m} \cdot\left(\frac{\partial v_{e}^{r}}{\partial y}\right) C_{n}
\end{array}\right\}
\end{aligned}
$$

$u_{e}^{s} S, v_{e}^{s} S$ and $u_{e}^{s} C, v_{e}^{s} C$ are the sine and cosine coefficients of the systematic error of the zonal and meridional wind fields, respectively. Similarly, $u_{e}^{r} S, v_{e}^{r} S$ and $u_{e}^{r} C, v_{e}^{r} C$ are same as above but for the random error. $u_{f} C, v_{f} C$ and $u_{f} S, v_{f} S$ are the cosine and sine coefficients, respectively for the zonal and meridional wind forecast. $k, m$ and $n$ in the subscripts represent three interacting wavenumbers in a triad. The Fourier coefficient of the derivative term is computed after taking the derivative of the respective variable.

\section{References}

Baumhefner D P and Downey P 1978 Forecast intercomparisons from three numerical weather prediction models; Mon. Weather Rev. 106 1245-1279.
Boer G J 1984 A spectral analysis of predictability and error in an operational forecast system; Mon. Weather Rev. 112 1183-1197.

Boer G J 1993 Systematic and random error in an extended range forecasting experiment; Mon. Weather Rev. 121 173-188.

Dalcher A and Kalaney E 1987 Error growth and predictability in operational ECMWF forecasts; Tellus 39A 474-491.

De S and Chakraborty D R 2004 Tropical systematic and random error energetics based on NCEP(MRF) analysisforecast system - A barotropic approach. Part II: In wavenumber domain; J. Earth Syst. Sci. 113 167-195.

De S and Chakraborty D R 2007 Variability of the tropical error characteristics in the NCEP(MRF) lower tropospheric wind forecasts during two contrasting seasons; Meteor. Atmos. Phys. 98 175-194.

Gall Robert, Blakeslee Richard and Somerville Richard C J 1979 Cyclone-scale forcing of Ultralong Waves; J. Atmos. Sci. 36 1692-1698. 
Hayashi Y 1979 A generalized method for resolving transient disturbances into standing and travelling waves by spacetime spectral analysis; J. Atmos. Sci. 36 1017-1029.

Heckley W A 1985 Systematic errors of the ECMWF operational forecast model in tropical regions; $Q$. J. R. Meteor. Soc. 111 709-738.

Kanamitsu M 1985 A study of predictability of ECMWF operational forecast model in the tropics; J. Met. Soc. Japan 63 779-804.

Kanamitsu M and Saha S 1995 Spectral budget of short range forecast error of NMC MRF model; Mon. Weather Rev. 123 1834-1850.

Krishnamurti T N, Subramanium M, Oosteroff D K and Daughenbaugh G 1990 Predictability of low frequency modes; Meteor. Atmos. phys. 44 63-83.

Krishnamurti T N, Chakraborty D R, Cubucku N, Stefanova L and Kumar T S V V 2003 A mechanism of the Madden-Julian Oscillation based on interactions in frequency domain; Q. J. R. Meteor. Soc. 129 2559-2590.

Krishnamurti T N and Chakraborty D R 2006 On the dynamics of phase locking; J. Atmos. Sci. 62 2952-2964.

Lambert S J and Merilees P E 1978 A study of planetary wave errors in a spectral numerical weather prediction model; Atmosphere-Ocean 16 197-211.

Lorenz E N 1969 The predictability of a flow which possesses many scales of motion; Tellus 21 289-308.

Lucarini Valerio, Calmanti Sandro, Dell'Aquila Alessandro, Ruti Paolo M and Speranza Antonio 2007 Intercomparison of the northern hemisphere winter mid-latitude atmospheric variability of the IPCC models; Clim. Dyn. 28 829-848.

Majda Andrew J and Biello Joseph A 2004 A multiscale model for tropical intraseasonal oscillations; Proc. Natl. Acad. Sci. USA 101 4736-4741.

Majda Andrew J and Stechmann Samuel N 2009 The skeleton of tropical intraseasonal oscillations; Proc. Natl. Acad. Sci. USA 106 8417-8422.

Murakami T and Tomatsu K 1964 The spectrum analysis of energy interaction terms in the atmosphere; J. Meteor. Soc. Japan 42 14-25.

Saltzman B 1957 Equations governing the energetics of the large scales of atmospheric turbulence in the domain of wave number; J. Meteor. 14 513-523.

Sikka D R and Gadgil S 1980 On the maximum cloud zone and the ITCZ over Indian longitudes during the southwest Monsoon; Mon. Weather Rev. 108 1840-1853.

Somerville Richard C J 1980 Topical influences on the predictability of the ultralong waves; J. Atmos. Sci. 37 $1141-1156$.

Straus D M and Shukla J 2005 The known, the unknown and the unknowable in the predictability of weather; $C O L A$ Technical Report, CTR 17520 pp.

Tomatsu K 1979 Spectral energetics of the troposphere and lower stratosphere; In: Advances in Geophysics (ed.) Saltzman B (New York: Academy Press) 21 289-401.

Tribbia J J and Baumhefner D P 2004 Scale interactions and atmospheric predictability: An updated perspective; Mon. Weather Rev. 132 703-713. 